\title{
Ammonia Production and Its Possible Role as a Mediator of Communication for Debaryomyces hansenii and Other Cheese-Relevant Yeast Species
}

\author{
K. Gori, ${ }^{1}$ H. D. Mortensen, N. Arneborg, and L. Jespersen \\ Department of Food Science, Food Microbiology, The Faculty of Life Sciences, University of Copenhagen, Rolighedsvej 30, \\ DK-1958 Frederiksberg C, Denmark
}

\section{ABSTRACT}

Ammonia production by yeasts may contribute to an increase in $\mathrm{pH}$ during the ripening of surface-ripened cheeses. The increase in $\mathrm{pH}$ has a stimulatory effect on the growth of secondary bacterial flora. Ammonia production of single colonies of Debaryomyces hansenii, Saccharomyces cerevisiae, Yarrowia lipolytica, and Geotrichum candidum was determined on glycerol medium (GM) agar and cheese agar. The ammonia production was found to vary, especially among yeast species, but also within strains of $D$. hansenii. In addition, variations in ammonia production were found between GM agar and cheese agar. Ammonia production was positively correlated to $\mathrm{pH}$ measured around colonies, which suggests ammonia production as an additional technological parameter for selection of secondary starter cultures for cheese ripening. Furthermore, ammonia appeared to act as a signaling molecule in $D$. hansenii as reported for other yeasts. On GM agar and cheese agar, D. hansenii showed ammonia production oriented toward neighboring colonies when colonies were grown close to other colonies of the same species; however, the time to oriented ammonia production differed among strains and media. In addition, an increase of ammonia production was determined for double colonies compared with single colonies of $D$. hansenii on GM agar. In general, similar levels of ammonia production were determined for both single and double colonies of $D$. hansenii on cheese agar.

Key words: Debaryomyces hansenii, ammonia production, signaling, cheese

\section{INTRODUCTION}

Surface-ripened cheeses are characterized by a ripening mediated by a smear consisting of a mixed popula-

Received November 10, 2006.

Accepted July 23, 2007.

${ }^{1}$ Corresponding author: klg@life.ku.dk tion of yeasts and surface bacteria, which contributes to the development of cheese flavor and texture due to its proteolytic and lipolytic activities (Brennan et al., 2004). The predominant yeast species observed during cheese ripening is Debaryomyces hansenii; however, strains of Saccharomyces cerevisiae, Yarrowia lipolytica, and Geotrichum candidum as well as a number of other yeast species have been reported (Fleet, 1990; Eliskases-Lechner and Ginzinger, 1995b; Jakobsen and Narvhus, 1996; Corsetti et al., 2001; Petersen et al., 2002). The growth of the less acid-tolerant bacteria including Brevibacterium linens, Corynebacterium spp., Arthobacter spp., Micrococcus spp., and Staphylococcus spp. is dependent on an increase in $\mathrm{pH}$ from about 5.0 to 5.8 or even $>6$ for some bacteria on the cheese surface (Seiler, 1986; Eliskases-Lechner and Ginzinger, 1995a; Valdés-Stauber et al., 1997). Generally, it is accepted that degradation of lactate in aerobiose to $\mathrm{CO}_{2}$ and $\mathrm{H}_{2} \mathrm{O}$ by the yeasts leads to an increase of $\mathrm{pH}$ (EliskasesLechner and Ginzinger, 1995b; Leclercq-Perlat et al., 1999), but production of alkaline metabolites such as ammonia may also be of importance (Brennan et al., 2004). However, the influence of ammonia production on the increase of $\mathrm{pH}$ has not been investigated to the same extent as lactate metabolism.

Quorum sensing is a well-known phenomenon by which microorganisms sense and respond to cell density-dependent signaling molecules (Miller and Bassler, 2001; Fuqua and Greenberg, 2002). Initial studies on quorum sensing have mostly focused on bacteria, and the phenomenon may be involved in growth of pathogenic and spoilage bacteria in foods such as meat products (Christensen et al., 2003; Bruhn et al., 2004), milk (Jay et al., 2003), and vegetables (Rasch et al., 2005). However, little is known about the role of quorum sensing of yeasts present in foods. In yeasts, quorum sensing has been described before; for example, in coordination of metabolism in S. cerevisiae at high cell densities with acetaldehyde as the signaling molecule (Richard et al., 1996); in stimulation of meiosis and sporulation in $S$. cerevisiae with bicarbonate as the signaling molecule 
(Hayashi et al., 1998; Ohkuni et al., 1998); and in prevention and stimulation of mycelial development in Candida albicans with farnesol and tyrosol as the respective signaling molecules (Hornby et al., 2001; Chen et al., 2004). Furthermore, ammonia has been reported to be involved in development and survival of neighboring colonies on agar plates (Palkova et al., 1997).

Produced ammonia has been found to be transmitted to neighboring yeast colonies, which responded by enhancing their own ammonia production, indicating that ammonia is a signaling molecule (Palkova et al., 1997). An increase in ammonia production has been found to lead to an alternative and more economical metabolism resulting in cell survival (Palkova and Forstova, 2000; Palkova et al., 2002; Palkova and Vachova, 2003). In addition, ammonia signaling typically results in asymmetric colonies forced to grow into the free space rich in nutrients. Several findings indicate that ammonia production involves amino acids released from the cellular stock or from protein degradation (Palkova et al., 1997; Zikanova et al., 2002). Ammonia has been reported as a general signaling molecule in yeasts. However, the role of ammonia as a signaling molecule involved in development and survival of colonies of $D$. hansenii highly relevant for cheese ripening has not yet been investigated.

In this study, the role of ammonia in the increase of $\mathrm{pH}$ on cheese surfaces was investigated by measurement of ammonia production of single colonies of different yeast species important for cheese ripening. Furthermore, it has been investigated whether strains of $D$. hansenii use ammonia for signaling and, thus, coordination of growth on both synthetic and cheese media.

\section{MATERIALS AND METHODS}

\section{Strains and Culture Conditions}

Three strains of Debaryomyces hansenii: D 18335, a dairy isolate (Petersen et al., 2001, 2002); MD 02 (Arla Innovation, Brabrand, Denmark); and the type strain of $D$. hansenii var. hansenii CBS 767 (Centraalbureau voor Schimmelcultures, Baarn and Delft, the Netherlands) were used in the present study. Furthermore, Saccharomyces cerevisiae D 7 (Gorgonzola isolate, University of Copenhagen, Denmark), type strains of Yarrowia lipolytica (CBS 2075, Centraalbureau voor Schimmelcultures), and Geotrichum candidum (CBS 615.84, Centraalbureau voor Schimmelcultures) were included in the study.

Yeast cultures were maintained at $-80^{\circ} \mathrm{C}$ in yeast glucose peptone (YGP) broth [per liter: $5 \mathrm{~g}$ of yeast extract (Difco Laboratories, Detroit, MI), $10 \mathrm{~g}$ of glucose (Merck, Darmstadt, Germany), and $5 \mathrm{~g}$ of Bacto peptone (Difco Laboratories), pH 5.6] containing 20\% (vol/vol) glycerol (Merck). Yeast cultures were propagated in 2 steps; first, YGP $(25 \mathrm{~mL})$ was inoculated with $1 \mathrm{~mL}$ of freeze culture and incubated for $48 \mathrm{~h}$ at $25^{\circ} \mathrm{C}$ with shaking at $120 \mathrm{rpm}$. Cells were counted, and $100 \mathrm{~mL}$ of YGP was inoculated with $1 \times 10^{6}$ cells per $\mathrm{mL}$, and incubated for $48 \mathrm{~h}$ under the same conditions as for the first propagation step, before the cultures were spotted onto agar plates.

\section{Determination of Ammonia Production}

Ammonia production was determined on glycerol medium (GM) agar [per liter: $10 \mathrm{~g}$ of yeast extract (Difco Laboratories), 3\% (vol/vol) glycerol (Merck), $30 \mathrm{mM}$ $\mathrm{CaCl}_{2}$ (Merck), and $20 \mathrm{~g}$ of agar (Difco Laboratories)] and on cheese agar. Cheese agar was prepared by autoclaving $20 \mathrm{~g}$ of Bacto agar (Difco) in $500 \mathrm{~mL}$ of demineralized water for $20 \mathrm{~min}$ at $121^{\circ} \mathrm{C}$, and then mixing it with $200 \mathrm{~g}$ of unsalted Danbo cheese and $12.5 \mathrm{~g}$ of trisodium citrate dihydrate (Merck) cooked in $400 \mathrm{~mL}$ of demineralized water for $1 \mathrm{~h}$ before use. To determine the influence of free amino acids on ammonia production, $0.5 \%$ casamino acids (Difco Laboratories) was added to the cheese agar. Before autoclaving, 0.01\% (wt/vol) of the $\mathrm{pH}$ indicator bromcresol purple (Sigma, St. Louis, MO) was added to both GM agar and cheese agar. The $\mathrm{pH}$ of both media was adjusted to 5.0, resulting in a dark yellow color of the GM agar and a bright green color of the cheese agar. Production of alkaline metabolites including ammonia changed the $\mathrm{pH}$ indicator to purple, whereas production of acidic metabolites changed the $\mathrm{pH}$ indicator to yellow. For determination of ammonia production of single colonies, 1 spot of $25 \mu \mathrm{L}$ of yeast culture $\left(1 \times 10^{7}\right.$ cells $)$ was spotted on the agar plates, whereas for determination of ammonia as a signaling molecule, 2 spots of $25 \mu \mathrm{L}$ of yeast culture $\left(1 \times 10^{7}\right.$ cells $)$ were spotted $1.5 \mathrm{~cm}$ apart on the agar plates, which subsequently were inverted. For absorption of volatile compounds produced by the growing colonies, a trap containing $200 \mu \mathrm{L}$ of $2 \%$ (wt/ vol) $\mathrm{HCl}$ was placed under the colonies. Plates were incubated at $25^{\circ} \mathrm{C}$ from d 0 to 8 . Every $24 \mathrm{~h}$, the 200 $\mu \mathrm{L}$ of $\mathrm{HCl}$ with absorbed volatile compounds was transferred to a 1.5-mL semimicro cuvette (Brand $\mathrm{GmbH}$ and Co, Wertheim, Germany) and $800 \mu \mathrm{L}$ of Nessler's reagent (Bie and Berntsen, Rødovre, Denmark) was added. After 30 min of incubation, the optical density $\left(\mathrm{OD}_{400 \mathrm{~nm}}\right.$ and $\left.\mathrm{OD}_{500 \mathrm{~nm}}\right)$ was measured using a Shimadzu UV-1201 spectrophotometer (SpectroChrom, Brøndby, Denmark). A standard curve using $\mathrm{NH}_{4} \mathrm{Cl}$ (Merck) was produced and used for calculation of the ammonia concentration. According to Barnes and Sugden (1990), linearity between $\mathrm{OD}_{400 \mathrm{~nm}}$ and the concentration of ammonia was determined in the range of 5 to $20 \mathrm{ppm}$, whereas 
linearity between $\mathrm{OD}_{500 \mathrm{~nm}}$ and the concentration of ammonia was determined in the range of 20 to $100 \mathrm{ppm}$. Colony diameters were measured and colony areas were calculated. The ammonia production of colonies was calculated as $\mathrm{ppm} / \mathrm{h}$ per $\mathrm{cm}^{2}$. All trials were performed in triplicate.

\section{pH Measurement}

Measurements of $\mathrm{pH}$ around yeast colonies were performed by the use of a surface electrode (InLab 426, Mettler-Toledo, Glostrup, Denmark) connected to a pH meter (1120, Mettler-Toledo). Adjustment of the electrode was performed with buffers with $\mathrm{pH} 4.01$ and 7.00 (Radiometer, Brønshøj, Denmark).

\section{Determination of Lactate Degradation}

Plugs with an area of $1 \mathrm{~cm}^{2}$, which corresponds to the area of the $\mathrm{pH}$ electrode, were taken out around single colonies on cheese agar. Four plugs were homogenized with $1 \mathrm{~mL}$ of water for 2 min using a stomacher. One milliliter of 1.7 $M$ TCA was added, and the suspension was homogenized for another $2 \mathrm{~min}$, and then incubated at $25^{\circ} \mathrm{C}$ for $1 \mathrm{~h}$. After incubation, the suspension was homogenized for another $2 \mathrm{~min}$ and filtered through a Millipore filter $(0.2 \mu \mathrm{m}$, Millipore, Billerica, MA). Lactate was measured by HPLC (HP series 1100, Hewlett-Packard, Palo Alto, CA) using a MicroGuard cation $\mathrm{H}$ cartridge followed by an Aminex $87 \mathrm{H}$ column (both from BioRad Laboratories, Hercules, CA). Lactate concentration was calculated as grams of lactate per gram of cheese agar.

\section{Determination of Casein Degradation}

Casein degradation was investigated on casein agar [per liter: $10 \mathrm{~g}$ of Hammerstein casein (Merck), $0.3 \mathrm{~g}$ of $\mathrm{Ca}(\mathrm{OH})_{2}$ (Merck), $0.2 \mathrm{~g}$ of $\mathrm{CaCl}_{2} \cdot 2 \mathrm{H}_{2} \mathrm{O}$ (Merck), and 15 $\mathrm{g}$ of agar (Difco Laboratories), $\mathrm{pH}$ 5.8]. One spot of 25 $\mu \mathrm{L}$ of yeast culture $\left(1 \times 10^{7}\right.$ cells $)$ was spotted on casein agar, and casein degradation was determined as clearing zones around colonies.

\section{Photography}

Colonies were photographed with a digital camera (EOS350D, Canon Inc., Tokyo, Japan) with and without transillumination. Photos with transillumination were used to visualize the orientation of ammonia production, whereas photos without transilluminations were used to visualize changes in colony morphology.

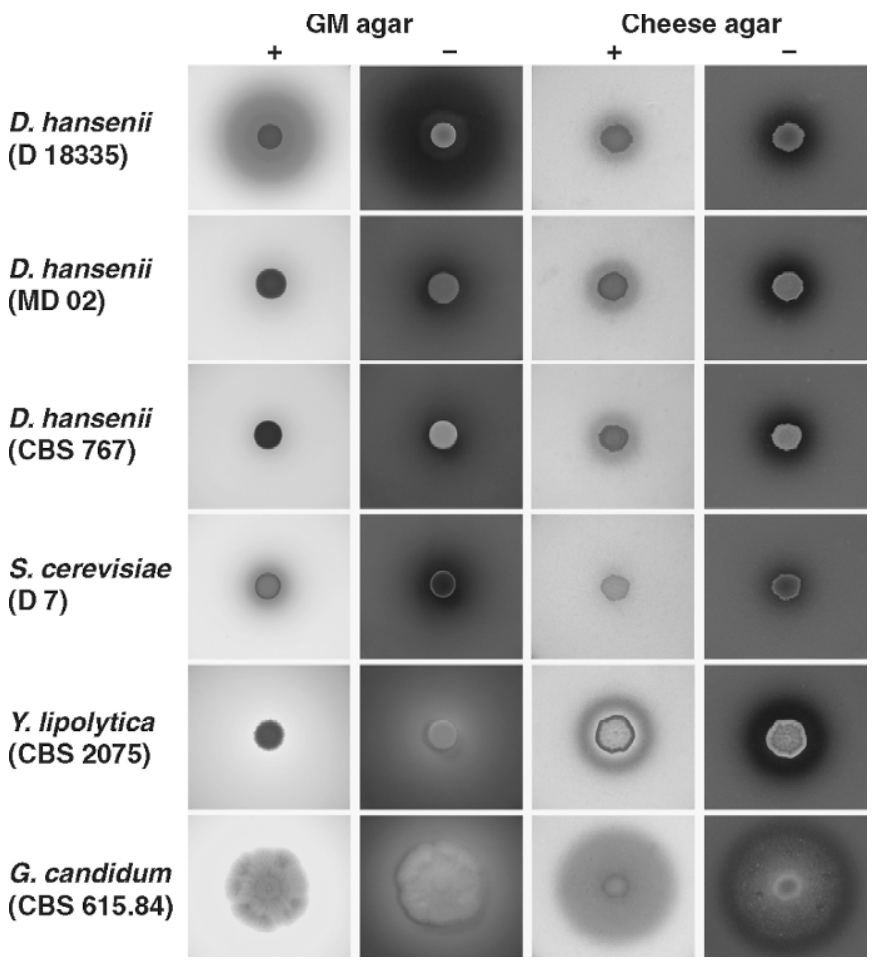

Figure 1. Ammonia production of single colonies of Debaryomyces hansenii (strains D 18335, MD 02, and CBS 767), Saccharomyces cerevisiae (D 7), Yarrowia lipolytica (CBS 2075), and Geotrichum candidum (CBS 615.84) determined on glycerol medium (GM) agar and cheese agar. Colonies were photographed after $4 \mathrm{~d}$ of incubation. Photos with transillumination were prepared to visualize changes in ammonia production $(+)$, whereas photos without transillumination were prepared to visualize changes in colony morphology (-). Color figure available at http://jds.fass.org/content/vol90/issue11/.

\section{RESULTS}

\section{Ammonia Production for Single Colonies}

Ammonia production was followed by color changes of the $\mathrm{pH}$ indicator bromcresol purple around single colonies of $D$. hansenii (D 18335, MD 02, and CBS 767), S. cerevisiae (D 7), Y. lipolytica (CBS 2075), and G. candidum (CBS 615.84) on glycerol medium (GM) agar and cheese agar, respectively (Figure 1). On GM agar, a shift from dark yellow to purple was observed for D. hansenii (D 18335, MD 02, and CBS 767) and $S$. cerevisiae (D 7), indicating that these yeast strains produced ammonia, whereas a shift from dark yellow to bright yellow was observed for Y. lipolytica (CBS 2075) and G. candidum (CBS 615.84), indicating that these yeast strains did not produce ammonia. The most intense purple color was observed for $D$. hansenii (D 18335). On cheese agar, a shift from bright green to purple indicating ammonia production was observed for all the investigated yeasts. The most intense purple color was observed for Y. lipolytica (CBS 2075). 

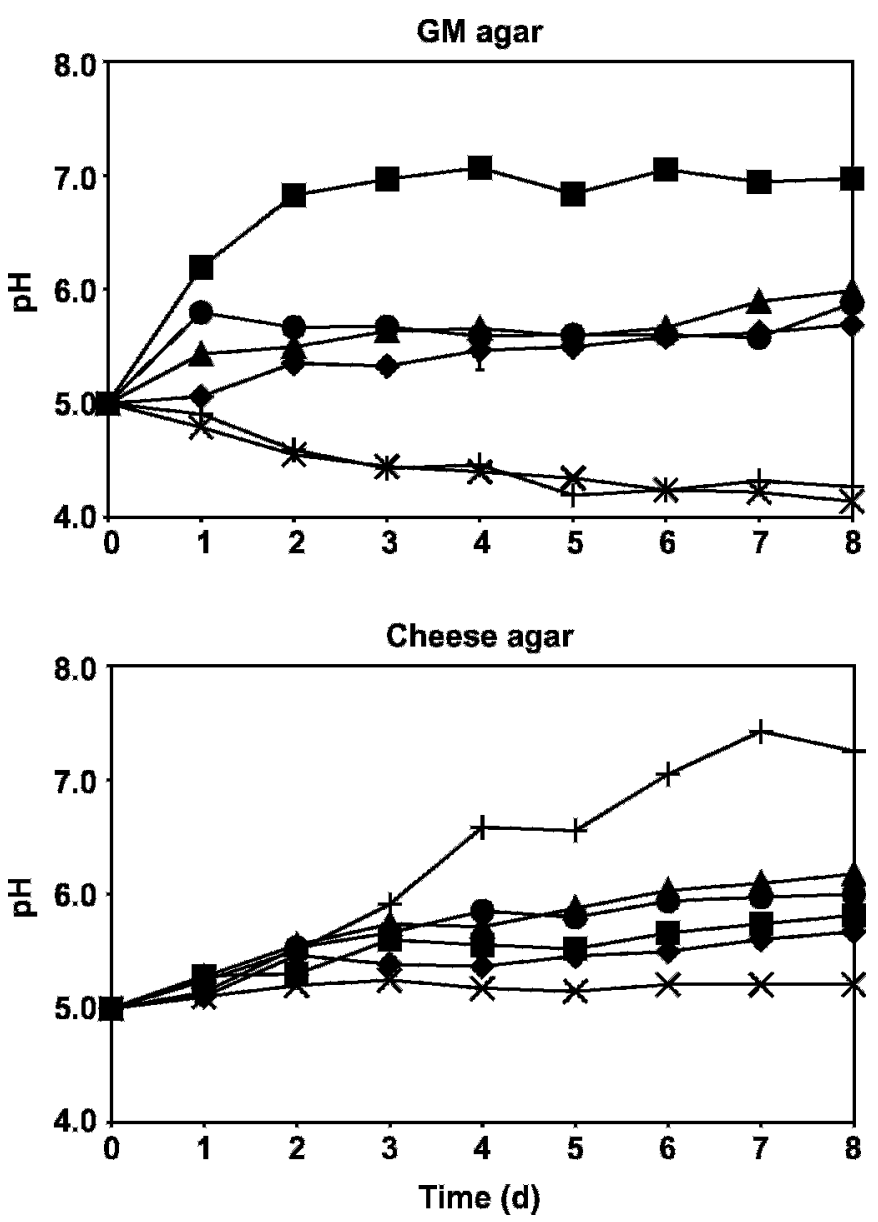

Figure 2. Measurement of $\mathrm{pH}$ around single colonies of Debaryomyces hansenii (strains D 18335, MD 02, and CBS 767), Saccharomyces cerevisiae (D 7), Yarrowia lipolytica (CBS 2075), and Geotrichum candidum (CBS 615.84) determined on glycerol medium (GM) agar and cheese agar. Yeast strains: $\mathbf{\square}=$ D. hansenii $(\mathrm{D} 18335) ; 0=$ D. hansenii (MD 02), $\mathbf{\Delta}=D$. hansenii (CBS 767), $\bullet=$ S. cerevisiae (D 7),$+=$ Y. lipolytica $($ CBS 2075), and $\times=$ G. candidum $($ CBS 684.15).

The $\mathrm{pH}$ was measured around yeast colonies to verify the effects observed by changes of the $\mathrm{pH}$ indicator (Figure 2). On both GM agar and cheese agar, a good correlation between the color changes of bromcresol purple and $\mathrm{pH}$ measurements was observed. On GM agar, the greatest increase of $\mathrm{pH}$ was observed around single colonies of $D$. hansenii (D 18335), for which $\mathrm{pH}$ increased from 5.0 to 6.8 during the first $2 \mathrm{~d}$, and remained constant at this value during the rest of the 8 $\mathrm{d}$ period. For D. hansenii (MD 02 and CBS 767) and $S$. cerevisiae (D 7), $\mathrm{pH}$ increased from 5.0 to an average value of 5.4 during $\mathrm{d} 1$ and 2 , and remained at this value. For Y. lipolytica (CBS 2075) and G. candidum (CBS 615.84), pH slightly decreased from 5.0 to 4.3 and 4.1 , respectively, during the investigated period. On cheese agar, the greatest increase in $\mathrm{pH}$ was observed around single colonies of $Y$. lipolytica (CBS 2075), for which $\mathrm{pH}$ slightly increased to 7.3 on $\mathrm{d} 8$. The $\mathrm{pH}$ around single colonies of $D$. hansenii (CBS 767 and MD 02) increased respectively to 6.2 and 6.0 on $\mathrm{d} 8$, whereas $\mathrm{pH}$ around single colonies of $D$. hansenii (D 18335) and S. cerevisiae (D 7) increased from 5.0 to slightly lower $\mathrm{pH}$ values (5.8 and 5.7, respectively). No changes in $\mathrm{pH}$ were observed around single colonies of $G$. candidum (CBS 615.84).

Ammonia production of single colonies of the included yeast strains was determined on both GM agar and cheese agar by Nessler's reagent. On GM agar, ammonia production was determined for $D$. hansenii (D 18335, MD 02, and CBS 767) and S. cerevisiae (D 7), whereas no ammonia production was detected for $Y$. lipolytica (CBS 2075) and G. candidum (CBS 615.84) (Figure 3). The greatest ammonia production on GM agar was determined for $D$. hansenii (D 18335). High ammonia production was noted particularly during the first $2 \mathrm{~d}$, when production of 6.3 and $6.6 \mathrm{ppm} / \mathrm{h}$ per $\mathrm{cm}^{2}$ was determined, respectively. Debaryomyces hansenii (MD 02 and CBS 767) and S. cerevisiae (D 7) produced similar levels of ammonia (between 0.72 and $1.9 \mathrm{ppm} /$ $\mathrm{h}$ per $\mathrm{cm}^{2}$ ) during the investigational period. On cheese agar, all the investigated strains produced ammonia (Figure 4), particularly Y. lipolytica (CBS 2075), which produced very high levels on cheese agar (unlike GM agar, in which no ammonia production was observed). The ammonia production of $Y$. lipolytica (CBS 2075) was $4.4 \mathrm{ppm} / \mathrm{h}$ per $\mathrm{cm}^{2}$ on $\mathrm{d} 1$, increased to $30 \mathrm{ppm} / \mathrm{h}$ per $\mathrm{cm}^{2}$ on d 5 , before it decreased to $9.5 \mathrm{ppm} / \mathrm{h}$ per $\mathrm{cm}^{2}$ on d 8. Debaryomyces hansenii (MD 02 and CBS 767) produced ammonia levels between 0.87 and $1.8 \mathrm{ppm} / \mathrm{h}$ per $\mathrm{cm}^{2}$, whereas D. hansenii (D 18335) and S. cerevisiae (D 7) produced slightly lower levels of ammonia (between 0.69 and $0.93 \mathrm{ppm} / \mathrm{h}$ per $\mathrm{cm}^{2}$ ). Geotrichum candidum (CBS 615.84) produced very low ammonia levels (between 0.042 and $0.30 \mathrm{ppm} / \mathrm{h}$ per $\mathrm{cm}^{2}$ ); however, the low value was primarily because of the increased colony size observed for this yeast strain. Without taking colony area into consideration, $G$. candidum (CBS 615.84) produced similar levels of ammonia as $D$. hansenii (D 18335) and S. cerevisiae (D 7) on cheese agar (results not shown).

Ammonia production for all strains of $D$. hansenii increased on cheese agar with the addition of $0.5 \%$ (wt/ vol) casamino acids (Figure 5). No significant differences could be observed among strains investigated. By d 1, an increase in ammonia production could be observed [465\% in average for D. hansenii (D 18335, MD 02, and CBS 767)] compared with cheese agar without addition of amino acids. On d 8, ammonia production was increased $[408 \%$ on average for $D$. hansenii (D 18335, MD 02, and CBS 767)], even though the level was slightly decreased compared with that on d $5[585 \%$ 

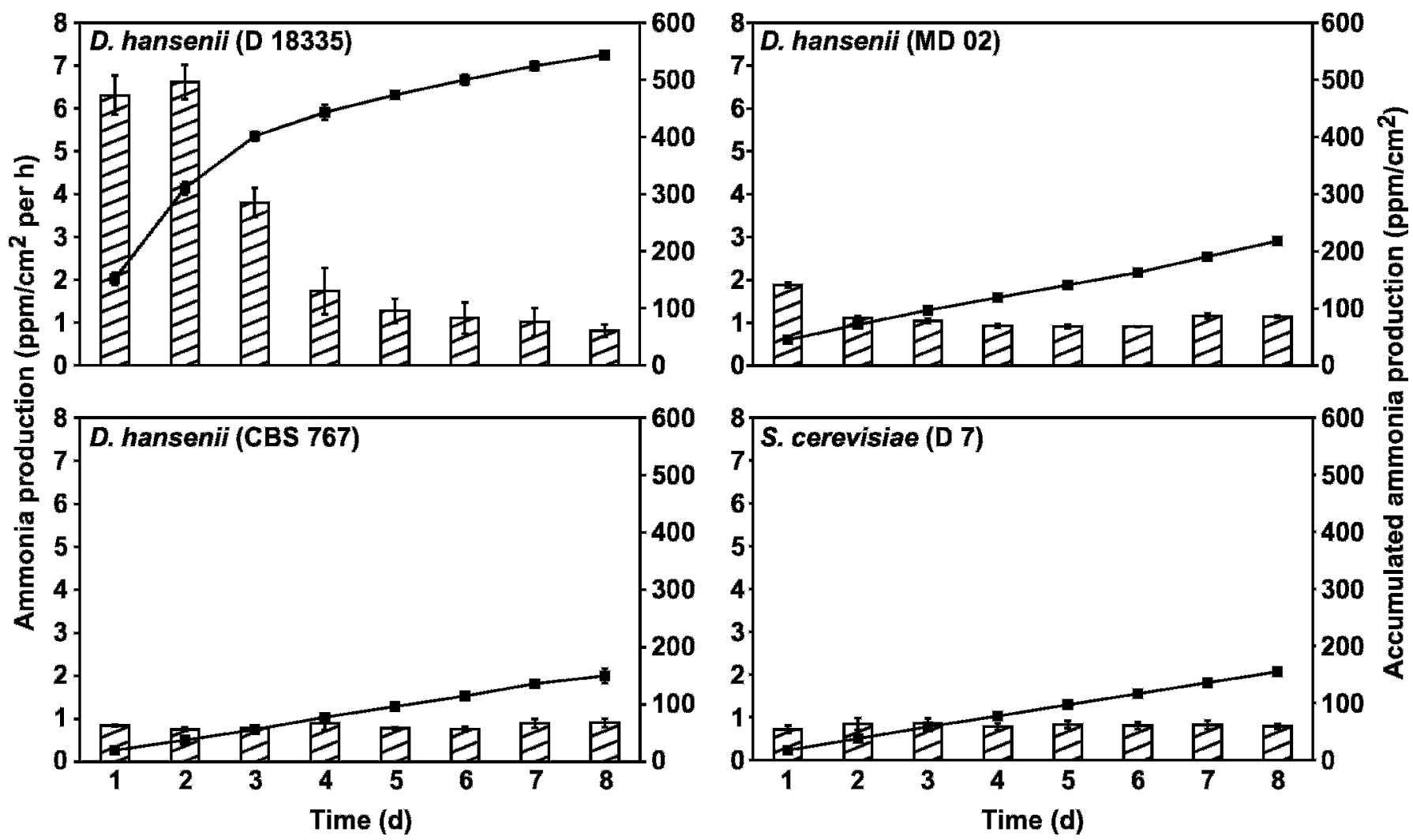

Figure 3. Ammonia production (bars) of single colonies of Debaryomyces hansenii (strains D 18335, MD 02, and CBS 767), Saccharomyces cerevisiae (D 7), Yarrowia lipolytica (CBS 2075), and Geotrichum candidum (CBS 615.84) determined on glycerol medium (GM) agar by Nessler's reagent; Y. lipolytica (CBS 2075) and G. candidum (CBS 615.84) did not produce ammonia on GM agar. The accumulated ammonia production is shown in curves.

on average for D. hansenii (D 18335, MD 02, and CBS 767)].

\section{Lactate Degradation}

Lactate degradation was determined in plugs excised around single colonies on cheese agar. For $D$. hansenii (D 18335, MD 02, and CBS 767), S. cerevisiae (D 7), and Y. lipolytica (CBS 2075), the level of lactate slightly decreased from 0.2 to $0.1 \mathrm{~g}$ of lactate per $100 \mathrm{~g}$ of cheese agar during the $8 \mathrm{~d}$ (data not shown). No significant lactate degradation was shown in plugs taken out around the colonies of the filamentous $G$. candidum (CBS 615.84).

\section{Ammonia Production for Neighboring Colonies}

The use of ammonia as a signaling molecule was investigated for D. hansenii (D 18335, MD 02, and CBS 767). Ammonia production was followed, when 2 colonies were spotted $1.5 \mathrm{~cm}$ apart on both GM agar and cheese agar (Figure 6). On GM agar and cheese agar, D. hansenii (D18335, MD 02, and CBS 767) showed ammonia production oriented toward neighboring colonies; however, the time to oriented ammonia production differed between strains and media. On GM agar, significantly oriented ammonia production between double colonies of $D$. hansenii (D 18335) was observed after $12 \mathrm{~h}$, whereas for D. hansenii (MD02 and CBS 767), it was observed after 16 and $20 \mathrm{~h}$, respectively. Unlike D. hansenii (MD 02 and CBS 767), for which ammonia was primarily oriented toward neighboring colonies, $D$. hansenii (D 18335) produced ammonia in directions other than toward neighboring colonies. On cheese medium, oriented ammonia production between double colonies was observed later than on GM agar. On cheese medium, significant oriented ammonia production between double colonies of $D$. hansenii (MD 02) was observed after $28 \mathrm{~h}$, whereas double colonies of $D$. hansenii (D 18335 and CBS 767) showed significantly oriented ammonia production after $36 \mathrm{~h}$. Asymmetric growth of the double colonies expanding to the free area was observed for D. hansenii (D18335, MD 02, and CBS 767) on cheese agar, unlike on GM agar.

Ammonia production for double colonies of $D$. hansenii (D18335, MD 02, and CBS 767) was determined 

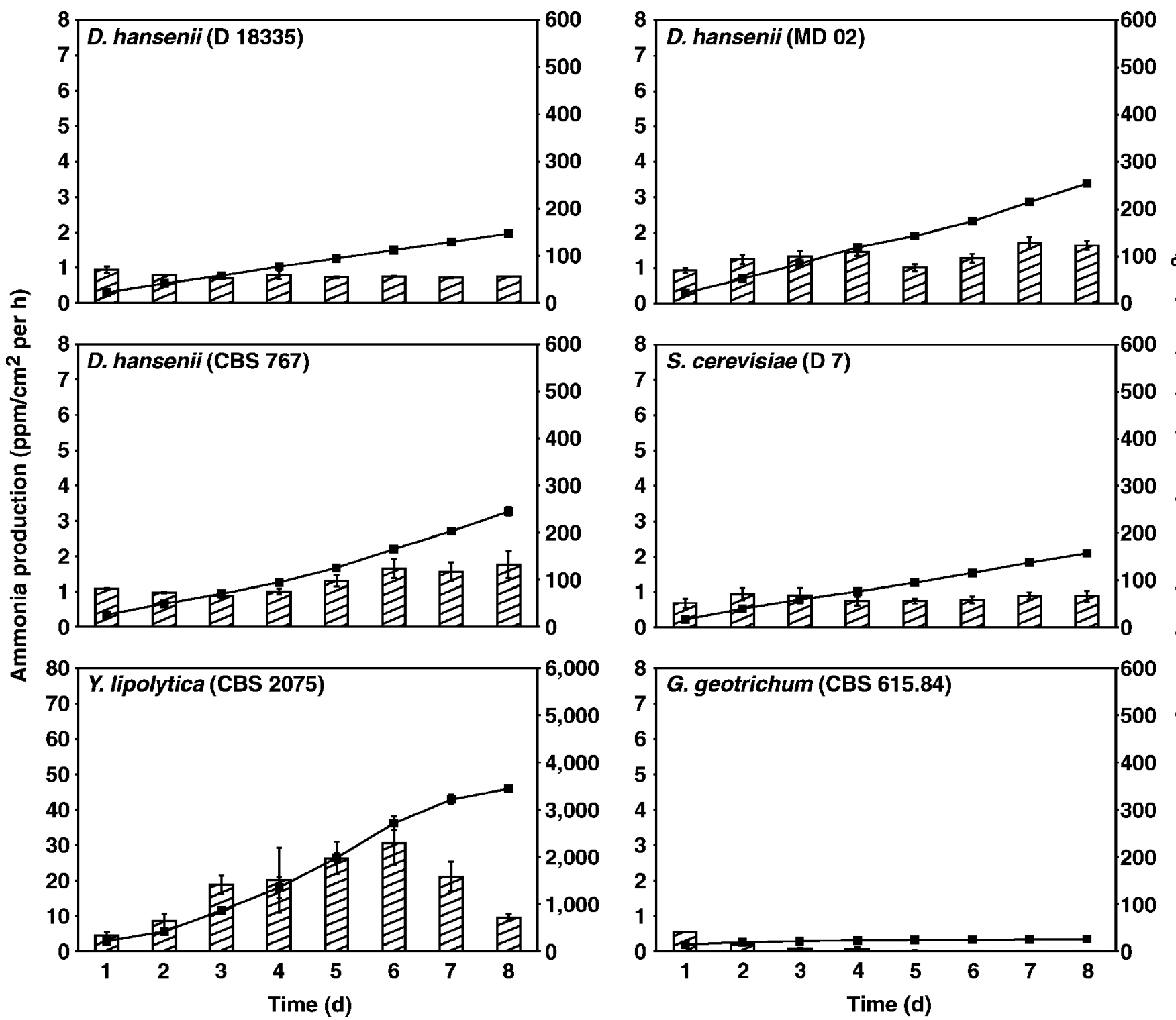

Figure 4. Ammonia production (bars) of single colonies of Debaryomyces hansenii (strains D 18335, MD 02, and CBS 767), Saccharomyces cerevisiae (D 7), Yarrowia lipolytica (CBS 2075), and Geotrichum candidum (CBS 615.84) determined on cheese agar by Nessler's reagent. The accumulated ammonia production is shown in curves. Note that the axes for the ammonia production and the accumulated ammonia production of Y. lipolytica (CBS 2075) are increased by a factor of 10 .

by Nessler's reagent, and ammonia production per colony and the percentage increase or decrease in ammonia production for double colonies compared with single colonies were calculated (Figure 7). On GM agar, the ammonia production of double colonies was greater than that obtained for single colonies of any $D$. hansenii strain. Indeed, a significant increase in ammonia production of double colonies compared with single colonies was observed from d 3 . For D. hansenii (D 18335), the increase in ammonia production was $27 \%$ on $\mathrm{d} 3$, and continued to increase ( $164 \%$ on $\mathrm{d} 6$ and $144 \%$ on $\mathrm{d}$
8). For D. hansenii (MD 02), the increase of ammonia production was $27 \%$ on $d 3$, it continued to increase to $77 \%$ on $\mathrm{d} 6$, and then it decreased to $17 \%$ on $\mathrm{d} 8$. For D. hansenii (CBS 767), the increase in ammonia production was $52 \%$ on d 3 , it continued to increase to $128 \%$ on $\mathrm{d} 6$, and then it decreased to $77 \%$ on $\mathrm{d} 8$. On cheese agar, an increase in the ammonia production for double colonies compared with single colonies was only seen for $D$. hansenii (MD 02) from d 4 to 6 , especially on d 5 , when an increase of $55 \%$ was obtained. Any increase in ammonia production for double colonies compared 


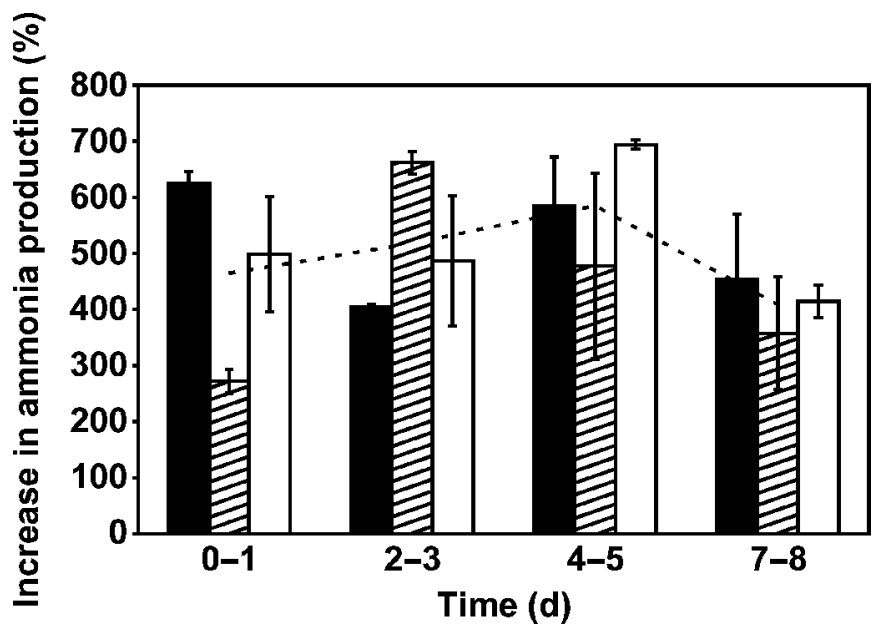

Figure 5. Increase in ammonia production (\%) by addition of $0.5 \%$ (wt/vol) casamino acids to cheese agar for Debaryomyces hansenii $\mathrm{D}$ 18335 (black bars); $D$. hansenii MD 02 (gray bars); and $D$. hansenii CBS 767 (white bars). The dotted line shows the average increase in ammonia production (\%) for D. hansenii (D 18335, MD 02, and CBS 767).

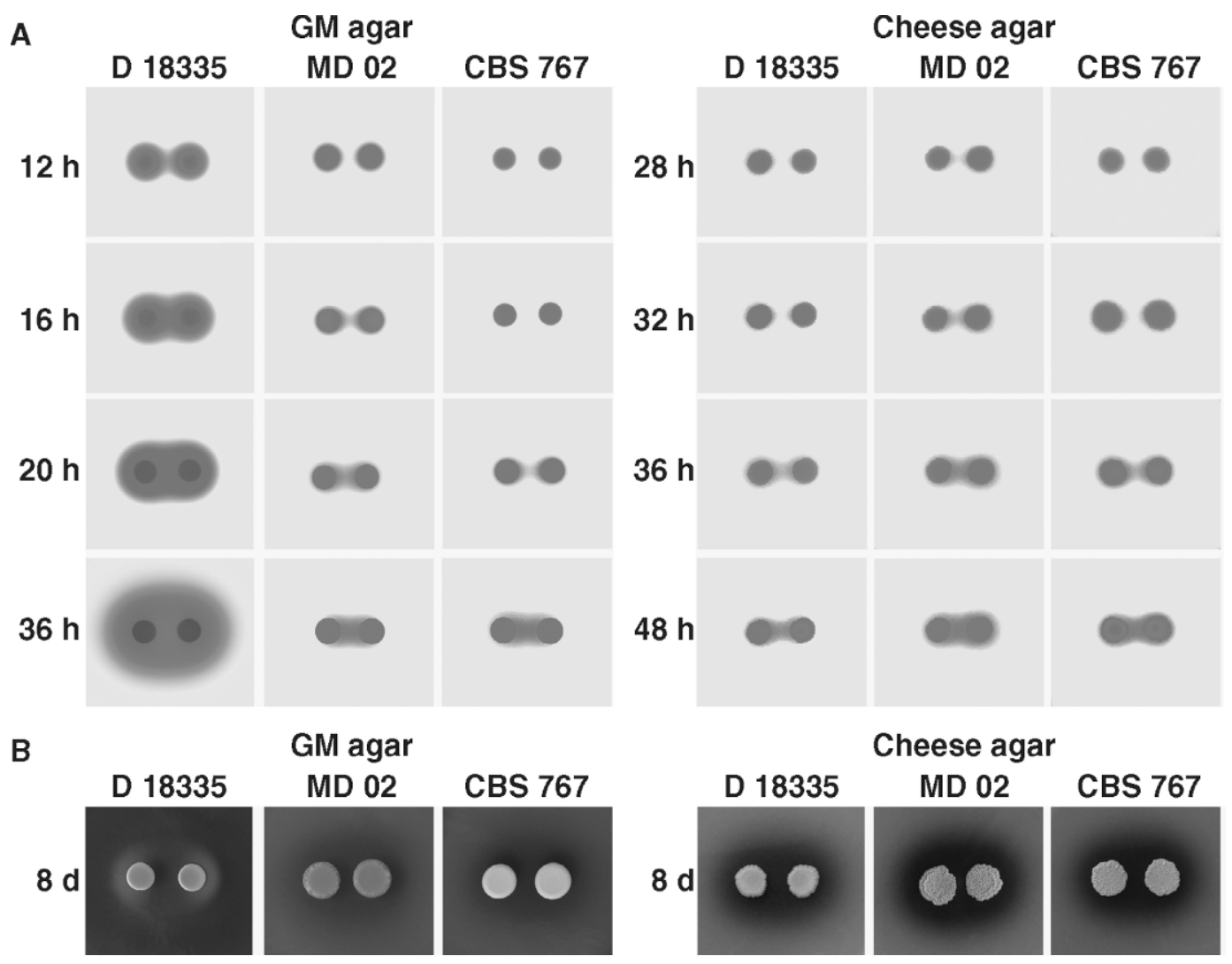

Figure 6. Ammonia production of double colonies of Debaryomyces hansenii (strains D 18335, MD 02, and CBS 767) on glycerol medium (GM) agar and cheese agar. Photos with transillumination (A) were prepared to visualize changes in ammonia production, whereas photos without transillumination (B) were prepared to visualize changes in colony morphology. Color figure available at http://jds.fass.org/content/ vol90/issue11/. 

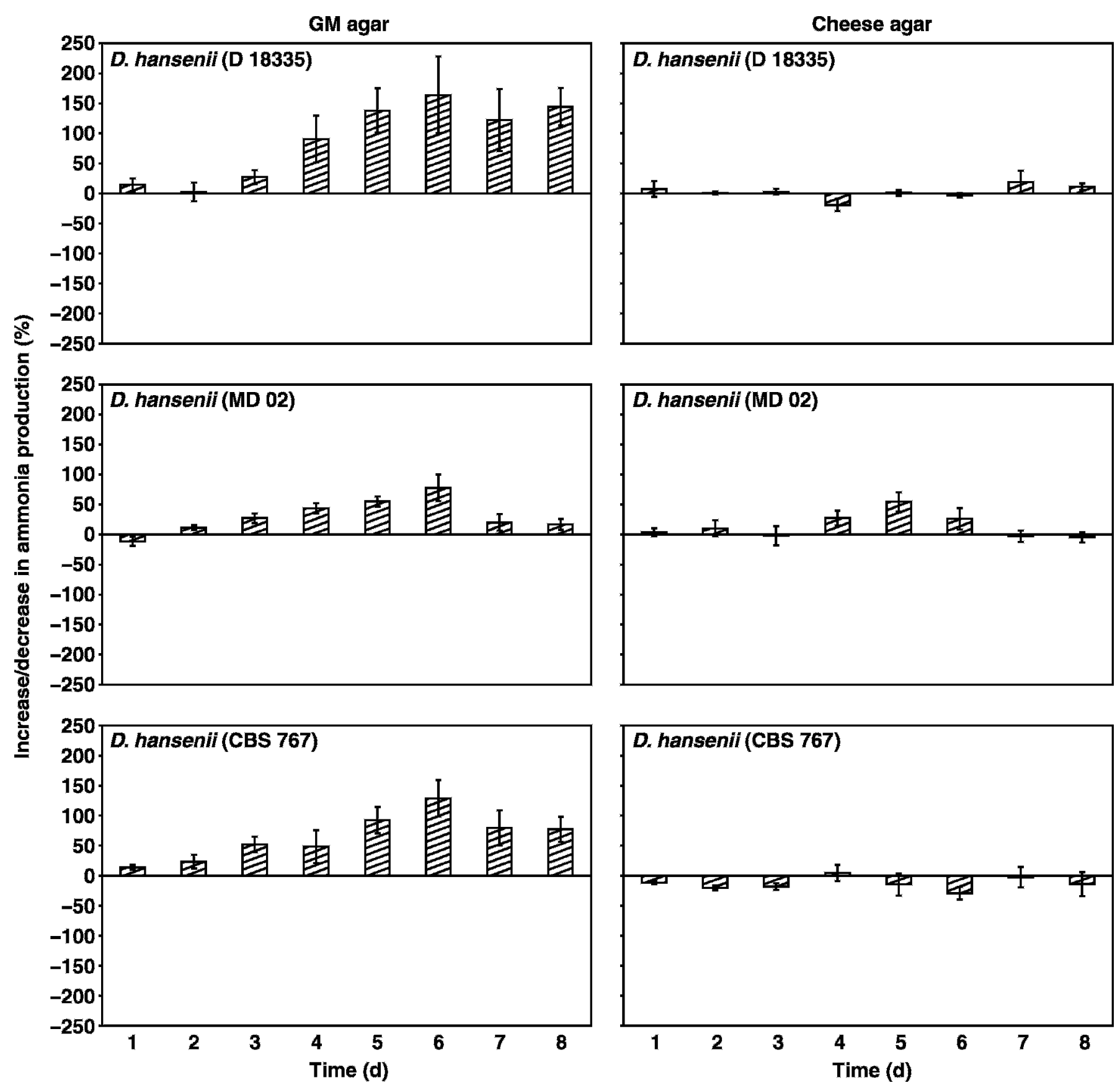

Figure 7. Increases and decreases (\%) in ammonia production of double colonies compared with single colonies for Debaryomyces hansenii (strains D 18335, MD 02, and CBS 767). The ammonia production for double colonies was calculated per colony.

with single colonies was observed for the other D. hansenii (D 18335 and CBS 767).

\section{DISCUSSION}

Ammonia production of the investigated yeasts was found to vary between species, but also among strains of $D$. hansenii. In addition, differences in ammonia pro- duction of the investigated yeasts were observed between GM agar and cheese agar. This is probably due to variations in the free amino acid content of the 2 media, because GM agar contains approximately $3 \mathrm{~g} / \mathrm{L}$ of free amino acids supplied from the yeast extract, whereas cheese agar contains only $0.3 \mathrm{~g} / \mathrm{L}$ of free amino acids (Ardö et al., 2002). Because only Y. lipolytica (CBS 2075) was able to degrade casein during the 8-d period 
(results not shown), casein degradation would not have contributed to ammonia production in D. hansenii (D18335, MD 02, and CBS 767), S. cerevisiae (D 7), or G. candidum (CBS 615.84) on cheese agar. Ammonia production for $S$. cerevisiae has been reported to be highly dependent on the availability of amino acids (Palkova et al., 1997; Zikanova et al., 2002). This was further confirmed in the present study for D. hansenii by addition of casamino acids to cheese agar (Figure 5). Furthermore, differences in ammonia production may be influenced by the ability of the investigated yeast strains to grow on glycerol, the carbon source in GM agar, or lactate, one of the main carbon sources in cheese agar.

The results showed that yeasts found on cheese surfaces produced ammonia indicating that ammonia production, as suggested by Brennan et al. (2004), may play a role in the increase of $\mathrm{pH}$ on cheese surfaces. A correlation between an increase of $\mathrm{pH}$ and ammonia production was observed for all yeast strains on both GM agar and cheese agar, indicating that differences in ammonia production are mainly responsible for these differences in $\mathrm{pH}$. Degradation of lactate has earlier been reported to play an important role in the increase of $\mathrm{pH}$ around colonies (Leclercq-Perlat et al., 1999). However, determination of lactate degradation in plugs excised around colonies grown on cheese agar showed that the lactate level decreased from 0.2 to $0.1 \mathrm{~g}$ per $100 \mathrm{~g}$ of cheese during the $8 \mathrm{~d}$ for all yeasts except of $G$. candidum. With respect to $G$. candidum, this study showed low levels of ammonia production (calculated per colony area) even though $G$. candidum is generally known for its deamination of amino acids resulting in ammonia production during cheese ripening (Hemme et al., 1982; Karahadian and Lindsay, 1987). In addition, no increase of $\mathrm{pH}$ around $G$. candidum colonies was determined. These observations may be biased due to the growth characteristics of $G$. candidum because ammonia is only involved in an increase of $\mathrm{pH}$ in the agar under the filamentous colony. Moreover, G. candidum is primarily used for flavor development, not to deacidify the cheese surface (Bockelmann et al., 2005). Based on the results obtained in the present study, ammonia production by yeasts may be taken into consideration as an additional technological parameter when starter cultures are selected for surface-ripened cheeses as previously described for other technological parameters such as $\mathrm{NaCl}$ tolerance (Gori et al., 2005) and adhesion (Mortensen et al., 2005).

Ammonia as a signaling molecule has been reported to be important for colony survival and development (Palkova et al., 1997). The signaling phenomenon is suspected to be general for yeasts. However, in this study, no ammonia production was determined for ei- ther $Y$. lipolytica (CBS 2075) or G. candidum (CBS 615.84 ) on GM agar earlier used to determine ammonia as a signaling molecule, indicating that ammonia is not involved in signaling in these yeast species. For the first time, this study showed that ammonia is involved in signaling in strains of $D$. hansenii. For D. hansenii (D 18335, MD 02, and CBS 767), ammonia was found to be oriented toward neighboring colonies when grown $1.5 \mathrm{~cm}$ apart on both GM agar and cheese agar, indicating that colonies of $D$. hansenii use ammonia as a signaling molecule for coordination of growth as reported for other yeast species (Palkova et al., 1997). In addition, ammonia production was greater for double colonies compared with single colonies of $D$. hansenii (D 18335, MD 02, and CBS 767) on GM agar. However, on cheese medium, ammonia production was only found to be greater for double colonies of $D$. hansenii (MD 02) from d 4 to 6 , even though a clearly oriented ammonia production could be observed on both GM agar and cheese agar. The deficiency in increased ammonia production on cheese agar may be because D. hansenii (D 18335, MD 02, and CBS 767) do not have sufficient proteolytic activity to degrade casein to free amino acids, which are responsible for ammonia production. Moreover, $D$. hansenii produces only endopeptidases (Klein et al., 2002). The cheese agar used will differ in several aspects from the environment on surface-ripened cheeses; for example, the composition of the microflora (and thus, the availability of different nitrogen sources) will be different. However, it is possible that ammonia production will increase for double colonies after $\mathrm{d} 8$.

Ammonia production is related to the colony structure such as observed for Candida mogii, in which ammonia changed the colony morphology from smooth to ruffled (Palkova and Forstova, 2000). In this study, $D$. hansenii (MD 02 and CBS 767) (the greatest ammonia production on cheese agar) showed ruffled colony morphology, whereas $D$. hansenii (D 18335) (the lowest ammonia production on cheese agar) showed smooth colony morphology. Ruffled colony morphology may be associated with longer lifetime of colonies due to more efficient water fluxes and removal of waste products compared with smooth colonies (Palkova et al., 2002).

In conclusion, ammonia production varied among yeast species and among different strains of $D$. hansenii and was influenced by the substrates used. Ammonia production plays an important role in the increase of $\mathrm{pH}$ on cheese surfaces, and may be taken into consideration as a technological parameter when starter cultures are selected for surface-ripened cheeses. Furthermore, ammonia apparently functions as a signaling molecule in $D$. hansenii as reported for other yeast strains. New knowledge about how $D$. hansenii communicate in complex environments such as a cheese sur- 
face will lead to increased information on how this important yeast species grows and proliferates in dairy products.

\section{ACKNOWLEDGMENTS}

This work is supported by the Danish Dairy Research Foundation (Danish Dairy Board), the Danish Ministry of Food, Agriculture and Fisheries, and the Centre for Advanced Food Studies (LMC). Rebecca Diana Houkjær is thanked for excellent technical assistance; Søren Lillevang (Arla Innovation) is thanked for providing $D$. hansenii (MD 02).

\section{REFERENCES}

Ardö, Y., B. V. Thage, and J. S. Madsen. 2002. Dynamics of free amino acid composition in cheese ripening. Aust. J. Dairy Technol. 57:109-115.

Barnes, A. R., and J. K. Sugden. 1990. Comparison of colourimetric methods for ammonia determination. Pharm. Acta Helv. 65:258-261.

Bockelmann, W., K. P. Willems, H. Neve, and K. H. Heller. 2005. Cultures for the ripening of smear cheeses. Int. Dairy J. 15:719-732.

Brennan, N. M., T. M. Cogan, M. Loessner, and S. Scherer. 2004. Bacterial surface-ripened cheeses. Pages 199-225 in Cheese: Chemistry, Physics and Microbiology. Vol. 2. P. F. Fox, P. L. H. McSweeney, T. M. Cogan, and T. P. Guinee, ed. Elsevier, Academic Press, Amsterdam, the Netherlands.

Bruhn, J. B., A. B. Christensen, L. R. Flodgaard, K. F. Nielsen, T. O. Larsen, M. Givskov, and L. Gram. 2004. Presence of acylated homoserine lactones (AHLs) and AHL-producing bacteria in meat and potential role of AHL in spoilage of meat. Appl. Environ. Microbiol. 70:4293-4302.

Chen, H., M. Fujita, Q. H. Feng, J. Clardy, and G. R. Fink. 2004. Tyrosol is a quorum-sensing molecule in Candida albicans. Proc. Natl. Acad. Sci. USA 101:5048-5052.

Christensen, A. B., K. Riedel, L. Eberl, L. R. Flodgaard, S. Molin, L. Gram, and M. Givskov. 2003. Quorum-sensing-directed protein expression in Serratia proteamaculans B5a. Microbiology-UK 149:471-483.

Corsetti, A., J. Rossi, and M. Gobbetti. 2001. Interactions between yeasts and bacteria in the smear surface-ripened cheeses. Int. J. Food Microbiol. 69:1-10.

Eliskases-Lechner, F., and W. Ginzinger. 1995a. The bacterial flora of surface-ripened cheeses with special regard to coryneforms. Lait 75:571-583.

Eliskases-Lechner, F., and W. Ginzinger. 1995b. The yeast flora of surface-ripened cheeses. Milchwissenschaft 50:458-462.

Fleet, G. H. 1990. Yeasts in dairy products. J. Appl. Bacteriol. 68:199-211.

Fuqua, C., and E. P. Greenberg. 2002. Listening in on bacteria: Acylhomoserine lactone signalling. Nat. Rev. Mol. Cell Biol. 3:685695.

Gori, K., H. D. Mortensen, N. Arneborg, and L. Jespersen. 2005. Expression of the GPD1 and GPP2 orthologues and glycerol retention during growth of Debaryomyces hansenii at high $\mathrm{NaCl}$ concentrations. Yeast 22:1213-1222.
Hayashi, M., K. Ohkuni, and I. Yamashita. 1998. An extracellular meiosis-promoting factor in Saccharomyces cerevisiae. Yeast 14:617-622.

Hemme, D., C. Bouillane, F. Metro, and M. J. Desmazeaud. 1982. Microbial catabolism of amino acids during cheese ripening. Sci. Aliments 2:113-123.

Hornby, J. M., E. C. Jensen, A. D. Lisec, J. J. Tasto, B. Jahnke, R. Shoemaker, P. Dussault, and K. W. Nickerson. 2001. Quorum sensing in the dimorphic fungus Candida albicans is mediated by farnesol. Appl. Environ. Microbiol. 67:2982-2992.

Jakobsen, M., and J. Narvhus. 1996. Yeasts and their possible beneficial and negative effects on the quality of dairy products. Int. Dairy J. 6:755-768.

Jay, J. M., J. P. Vilai, and M. E. Hughes. 2003. Profile and activity of the bacterial biota of ground beef held from freshness to spoilage at $5-7^{\circ} \mathrm{C}$. Int. J. Food Microbiol. 81:105-111.

Karahadian, C., and R. C. Lindsay. 1987. Integrated roles of lactate, ammonia, and calcium in texture development of mold surfaceripened cheese. J. Dairy Sci. 70:909-918.

Klein, N., A. Zourari, and S. Lortal. 2002. Peptidase activity of four yeast species frequently encountered in dairy products-Comparison with several dairy bacteria. Int. Dairy J. 12:853-861.

Leclercq-Perlat, M. N., A. Oumer, J. L. Bergere, H. E. Spinnler, and G. Corrieu. 1999. Growth of Debaryomyces hansenii on a bacterial surface-ripened soft cheese. J. Dairy Res. 66:271-281.

Miller, M. B., and B. L. Bassler. 2001. Quorum sensing in bacteria. Annu. Rev. Microbiol. 55:165-199.

Mortensen, H. D., K. Gori, L. Jespersen, and N. Arneborg. 2005. Debaryomyces hansenii strains with different cell sizes and surface physicochemical properties adhere differently to a solid agarose surface. FEMS Microbiol. Lett. 249:165-170.

Ohkuni, K., M. Hayashi, and I. Yamashita. 1998. Bicarbonate-mediated social communication stimulates meiosis and sporulation of Saccharomyces cerevisiae. Yeast 14:623-631.

Palkova, Z., F. Devaux, M. Ricicova, L. Minarikova, S. Le Crom, and C. Jacq. 2002. Ammonia pulses and metabolic oscillations guide yeast colony development. Mol. Biol. Cell 13:3901-3914.

Palkova, Z., and J. Forstova. 2000. Yeast colonies synchronise their growth and development. J. Cell Sci. 113:1923-1928.

Palkova, Z., B. Janderova, J. Gabriel, B. Zikanova, M. Pospisek, and J. Forstova. 1997. Ammonia mediates communication between yeast colonies. Nature 390:532-536.

Palkova, Z., and L. Vachova. 2003. Ammonia signaling in yeast colony formation. Int. Rev. Cytol. 225:229-272.

Petersen, K. M., P. L. Møller, and L. Jespersen. 2001. DNA typing methods for differentiation of Debaryomyces hansenii strains and other yeasts related to surface ripened cheeses. Int. J. Food Microbiol. 69:11-24.

Petersen, K. M., S. Westall, and L. Jespersen. 2002. Microbial succession of Debaryomyces hansenii strains during the production of Danish surfaced-ripened cheeses. J. Dairy Sci. 85:478-486.

Rasch, M., J. B. Andersen, K. F. Nielsen, L. R. Flodgaard, H. Christensen, M. Givskov, and L. Gram. 2005. Involvement of bacterial quorum-sensing signals in spoilage of bean sprouts. Appl. Environ. Microbiol. 71:3321-3330.

Richard, P., B. M. Bakker, B. Teusink, K. van Dam, and H. V. Westerhoff. 1996. Acetaldehyde mediates the synchronization of sustained glycolytic oscillations in populations of yeast cells. Eur. J. Biochem. 235:238-241.

Seiler, H. 1986. Identification of cheese smear coryneform bacteria. J. Dairy Res. 53:439-449.

Valdés-Stauber, N., S. Scherer, and H. Seiler. 1997. Identification of yeasts and coryneform bacteria from the surface microflora of brick cheeses. Int. J. Food Microbiol. 34:115-129.

Zikanova, B., M. Kuthan, M. Ricicova, J. Forstova, and Z. Palkova. 2002. Amino acids control ammonia pulses in yeast colonies. Biochem. Biophys. Res. Commun. 294:962-967. 